\title{
Using digitally distributed vulgar comedy to reach young men with information about healthy sexual development
}

\author{
Alan McKee, Anthony Walsh and Anne-Frances Watson
}

\begin{abstract}
Focus groups show that young men do not have available to them the same resources to learn about healthy sexual development as do young women. A collaborative project led by a leading provider of sexuality education aimed to reach young men with information about healthy sexual development by using a genre that focus groups showed they favour - vulgar comedy. This project raised two important issues. Firstly, comedy is ambivalent - it is by definition not serious or worthy. This challenges health communication, which traditionally favours the clear presentation of correct information. Secondly, vulgarity can be challenging to the institutions of health communication, which can be concerned that it is inappropriate or offensive. This article addresses these issues and reports on the materials that emerged from the project.
\end{abstract}

\section{Keywords}

Entertainment education, young men, boys, comedy, sexual health, vulgar

\section{Introduction}

This article reports on the use of digitally-distributed vulgar comedy videos as a way to reach young men with information about healthy sexual development. As part of a project aiming to find out why young people do not always practice safe sex, a series of focus groups with 14-16 year olds in Brisbane explored what they know about sex and how they found it out. The data showed that while young women have easy access to media that explore issues of healthy sexual development - such as Girlfriend and Dolly magazines - young men's media consumption of sport, video games, music and vulgar comedy such as South Park does not currently offer such a cultural space. This gap is addressed by a project 
developed by Family Planning Queensland that aims to reach young men by creating vulgar comedy about sexual health issues that can reach them through their favoured media consumption channels such as YouTube.

\section{Understanding young men's cultures of sexual learning}

This project aimed to develop improved materials for reaching young people with information about healthy sexual development. Taking a 'culture-centered' approach to health communication (Dutta 2008) we started by listening to young people, aiming to 'identif[y] problems and accompanying solutions from within the culture' (Dutta 2008, 255). We conducted twenty focus groups with eighty-nine young people between the ages of fourteen and sixteen from five Brisbane schools in order to find out which sources they used to get various kinds of information about sex, including formal schooling, parents, peers and the media (for full details about the research method see McKee, Dore, and Watson 2014).

This data revealed that young men are poorly served with information about healthy sexual development. Across all sources of information - school, family, peers and the media - there were clear, gendered differences in the young people's sexual learning. Learning from sex education at school differed by gender.

3.M.1 Yeah, the schools kind of promote, you know, say no if you don't want to do it. Except they don't really promote it for guys, they more promote it for girls...

Similarly, experiences of learning from parents about sex differed by gender. Young men would be the target of 'jokes' from parents, particularly fathers:

3.M.2 Yeah, my dad's like 'Do you want to cut some fingers off a glove before you go?' [to use as a condom] and I'm like 'No' (laughing).

By contrast parents treated the possibility of young women having sex as something very serious. Young people's learning about sex from peers was also gendered. Young women had a culture of talking openly about relationships. By contrast the young men in our focus groups gave little sense that they had an 
open culture of discussion about sexuality and relationships. When asked what was the best way to get what you want in a relationship, one group of young men said:

6.M.1: It's not really a topic we discuss much.

6.M.2: Yeah, you wouldn't talk about it with your friends or anything really.

Given that learning about sex seems to be so gendered it is not surprising that the ways in which young men learned about sex from the media also proved to be different from young women's. The young women in our focus groups had a culture of consuming entertainment resources that address issues about sexuality and relationships, and then using these as a way to talk about these issues with peers, mentioning magazines such as Girlfriend and Dolly. By contrast members of our male focus groups did not consume any magazines - perhaps surprisingly, not even 'lads mags' like Zoo Weekly, People or Picture. Some of the young men did consume pornography, although this was not volunteered in discussions and the facilitator had to work hard to create a safe space where they could acknowledge their consumption. This was not a large part of the focus group discussions. The forms of entertainment that the young men talked most about were social media (Facebook, YouTube), computer games (Call of Duty, Halo), sport, pop music (rap) and comedy films and television. This last group of texts was particularly interesting for our project. They did not watch 'romantic comedy' films - the examples given in the focus groups were Harold and Kumar Go To Whitecastle, Harold and Kumar Go To Guantanamo Bay, South Park, Family Guy, Entourage, Californication and Angry Boys. We believe that this group of texts share a characteristic - they are all vulgar comedies, and this makes them interesting for a project seeking to reach young men with sexual information. In this context use the term 'vulgar' along three axes. The first meaning is 'common': the Oxford English Dictionary definition of 'vulgar' includes 'Of persons: Belonging to the ordinary or common class in the community; not distinguished or marked off from this in any way; plebeian'. That is to say, 'vulgar' language is - literally - the language of ordinary people. The second meaning of vulgar we wish to draw upon is the everyday implication that 'vulgar' 
culture deals with reproductive and/or alimentary topics - having sex, farting, defecating, and so on. The third meaning of vulgar is equivalent to 'rude' - the use of swearwords and disrespect for propriety. These terms are closely linked it is not coincidental that one literal meaning of 'rude' in the OED is 'uneducated'. Common, uneducated, swearing and interest in sex - this class-based constellation of meanings circulate around vulgarity. And we believe that this makes vulgar comedy an effective modality for sexual health messages (see Byron, Albury, and Evers 2013).

\section{The uses of comedy}

Do young men learn from vulgar entertainment? It is certainly possible to argue that vulgar comedy speaks to young men about sexuality - by definition this is part of what makes it 'vulgar' (in the sense of 'related to sex'). However the young men did not see vulgar comedy as educational in any simple way. They stated that although issues around sex were mentioned in the comedies, a consumer wouldn't learn anything from watching it - precisely because of its generic status as comedy:

Facilitator:

Has South Park or Family Guy ever said anything about these kind of things?

3.M.1 Once or twice, yeah.

Facilitator: So what kind of stuff?

3.M.1 They don't really promote it in any of the TV shows that I watch. They kind of like make fun of it.

The fact that the programs make 'fun' is presented as evidence that they don't 'promote' learning about sex or relationships. Another young man makes a similar point when he suggests that:

3.M.2 No. Um, in South Park you don't really learn anything. It's just kind of (laughing) yeah.

The question of how vulgar comedy might work as a source of sexuality and relationships education is clearly a complex one. The genre of comedy is often 
ambivalent and contains contradictory messages, and theorists have long disagreed about how it might communicate information. Does comedy undermine what it makes fun of, or - by representing it - support it? George Orwell asserts that 'whatever is funny is subversive' (quoted in Palmer 1987, 11). By contrast Julia Kristeva argues that humour is conservative because it is 'the law anticipating its own trangression' (quoted in Hutcheon 1985). We tend to agree with the work of Jerry Palmer, who argues that neither of these positions is entirely satisfactory: indeed, comedy is 'simultaneously conservative and subversive' (Palmer, 1987: 14). In order for comedy to work there must always be simultaneous and perhaps contradictory responses to a text. If an implausible element is not present, cuing the reader to dismiss it as being ridiculous, then the text is no longer comedy - it becomes 'serious'. But at the same time if there is no plausible element present in the text then it is not possible to make any sense of it and it slips towards nonsense. This means that:

We cannot conclude a priori that humour in general is harmless or oppressive ... because humour is a contextual phenomenon we need to see how given jokes function in particular situations (Lewis 1989, 39)

The data from our focus groups allows us to explore this issue. To start at the simplest level we can state with certainty that young men remember ideas about sex that they have encountered in the form of vulgar comedy. When we asked them to talk about what they knew about a number of sexual issues they would bring up examples from vulgar comedy. Talking about how to ask people out or break up with them one young man recalled:

4.M.2 Um, I've seen one thing. But then it's just, ah like one of the comedy shows. Take them to a public place so they don't like get angry, because they don't want to look like idiots in front of a lot of people.

Comedy YouTube videos about issues relating to relationships and sexuality were also discussed. For one group this arose as they were talking about how to say 'no' to sexual advances you don't want:

6.M.1: Well, there's a YouTube video called Mr Teddy, because he's saying when a girl has sex with the guy and it's like the girl's choice basically 
because the guy's - he's saying that a guy is a definite 'yes', and it's because the guy's a legend if he has sex and the girl's a slut because it was her choice or something. So that - and he's somewhere else in Australia, so that's sort of a broad message.

So we can state with certainty that, despite their comments about the lack of 'learning' from comedy, young men can - and do - recall information from comedic sources when discussing what they know about various aspects of sexuality and relationships. What is less clear is how this information is taken up in their lives and if it becomes part of their own practice. As we noted above, information about sexuality or relationships in a magazine like Girlfriend might serve to provoke peer discussions of issues for girls. By contrast, while there was some evidence in the focus groups that content from comedy was transferred into everyday practice, the ways in which this happened were not predictable. A discussion in one focus group about 'Asking for what you want in a relationship' led to a conversation about different kinds of sexual relationships - and this led to a discussion of Quagmire, a character from the comedy Family Guy who is radically sexually promiscuous and has little respect for consent:

4.M.2 They're shown to be like something that you should like strive for. Like Quagmire for instance, like he's set up to be like a real player character who gets like every girl. And like the audience is viewed to be like 'Oh I wish I was like Quagmire'.

4.M.5 I kind of think my position to view him negatively because he's just a, a seedy... like he's been everywhere... like if you know what I mean.

4.M.2 Yeah, I was just thinking the whole positive thing because like everyone uses that whole 'Giggity-giggity' thing.

4.M.5 Yeah.

4.M.2 Like a catch phrase. Like everyone, whenever like anyone like says anything sexual, people will be like 'Giggity-giggity'. Even on Facebook, it's just like an extra comment after anything that could be taken dirty, just 'Giggity'. 
'Giggity giggity' is Quagmire's catchphrase, used whenever he is thinking about sex - often to signal a possible double-entendre in a conversation, in the same way that members of an older generation might use 'that's what she said' or '...as the actress said to the bishop'. We can see that young people are using it playfully to create vulgar humour in their interactions - but it's less clear how this is valued. In this focus group there is disagreement about whether this profoundly amoral and sexually-obsessed character is someone you should 'strive for' or if he should be dismissed as 'seedy'. Even without making a final judgment on this point we can say with certainty that this catchphrase has become part of a process of double-entendre whereby young people playfully bring sex into everyday conversations.

While the young men insisted that they did not 'learn' about sexuality from this comedy, then, they nevertheless recalled information that they had heard from comedy, and applied it in their own lives - although not in straightforward ways. Even if it is difficult to navigate the ways in which vulgar comedy contributes to young men's sexual learning, we believe, if we are seeking to reach young men with information about healthy sexual development then vulgar comedy can play a role.

\section{Challenge 1: not being 'worthy'}

Blokes Talking is a project initiated by Family Planning Queensland to create short vulgar comedy videos which can be distributed online to reach young men with sexual health information. These videos involve male stand-up comedians telling jokes about sexual-health topics as a way to promote discussion of these issues by young men.

FPQ recruited eleven male comedians ranging in age from teens to late 50s. They were asked, on camera, a series of questions designed to prompt jokes across a range of sexual health topics. The questions they were asked were developed by an Advisory Group of education and health experts to allow discussion of topics across the range of factors identified as relevant to healthy sexual development by McKee et al. (2010). These include, as well as 'Education about biological 
aspects of sex', a range of skills and competencies such as 'An understanding of consent', 'Relationship skills', 'Awareness and acceptance that sex can be pleasurable' and 'Competence in mediated sexuality'. An iterative process was used whereby possible questions were developed relating to the domains of healthy sexual development and then the Advisory Group gave feedback. During this process it became clear that at least two quite different imperatives were in place.

Some members of the Advisory Group were keen that the questions should try to ensure that the comedians communicated sexual health information as accurately as possible, and in ways that matched the priorities of sexual health experts. But at the same time, other members of the Advisory Group wanted the questions to be formulated in such a way that they maximized the entertainment value of the responses and avoided the danger of being 'worthy' or 'preachy' - as it is known that either of these qualities turn off a youth audience. So, for example, an early draft of the questions asked the comedians 'How do you feel about homophobia?'. The language used in this question is formal, and it is worded in such a way as to allow only one answer (nobody would say 'I think it's great'). Following discussions about maximizing the entertainment value of the material, and minimizing preachiness, the question evolved into: 'What's your reaction if a bloke cracks onto you? (or if you're gay, what do you do if a woman cracks onto you?)' . This allowed the comedians to use personal experiences for humour, and moved away from abstract condemnations of homophobia.

This example points to a wider tension in entertainment education.

Entertainment education (E-E) - 'the intentional placement of educational content in entertainment messages' - is a growing area in health communication (Singhal and Rogers 2002,117), and one that works well with a culture-centred approach. Entertainment education recognizes that audiences are not keen to be lectured to by institutionalized experts and often resist such messages (a response named 'reactance' in the literature) (Moyer-Guse and Nabi 2010, 29). However, much research on entertainment education still takes an adversarial approach, whereby target audiences are seen as opponents whose 'resistance' is to be 'overcome' (Moyer-Guse and Nabi 2010). It is still assumed that the 
message content will be generated by experts, who will then try to find ways to force it onto target populations. There are fundamental problems with this approach. David Buckingham and Sara Bragg found from their interviews with young people that they were likely to reject worthy programs that they saw as 'preaching' to them (Buckingham and Bragg 2004, 162). Indeed:

the overt imposition of moral lessons .... is precisely [the] kind of approach that leads some viewers to perceive [entertainment] as preaching and lecturing and to reject [its messages] on these grounds (Buckingham and Bragg 2004, 168).

By contrast, entertainment products (Buckingham and Bragg take the example of soap operas) can lead young people to work things out for themselves: they 'encourag[e] viewers to make their own judgments, rather than simply commanding their assent' (Buckingham and Bragg 2004, 168). This engages young people and encourages deep learning. But, the challenge of these forms of communication is that the more they engage young people, the less likely they are to have a single clear 'message' that all viewers will agree on. The soap opera stories that the young people in Buckingham and Bragg's research were most engaged by, remembered best, and discussed with most passion also led to disagreements between them about what message they actually communicated (as with the example of Quagmire in our focus groups) (Buckingham and Bragg $2004,174)$. This point bears some emphasis for it is a key challenge for health communicators: the very characteristic that makes a text powerful for pedagogy - the fact that it offers grey areas for discussion - also means that its message must be less clear cut (less 'preaching'). As health communicators we can feel the urge to ensure that young people only receive the correct information. But this doesn't allow them to make up their minds for themselves, and runs the risk of being seen as preaching and as a result, less effective, or indeed, totally ignored. It can be difficult for us to embrace approaches that might give young people a range of perspectives and allow them to reach their own decisions about what is best for them.

\section{Challenge 2: embracing vulgarity}


As well as this move away from 'preaching', entertainment education for young men raises another challenge - one of taste. The young men in our focus groups like vulgar comedy. This can be difficult for serious and well-intentioned educators who might not enjoy such forms of culture, and indeed may be confronted by them. In the focus groups we made a concerted effort to get young people to talk in their own language and not to present the typical performance that they know adults usually expect of them:

Facilitator: First thing, be as rude as you like ... I don't want to know what you would tell your parents. I don't want to know what you would tell your teachers. I want to know the truth. So be honest, that's, otherwise it doesn't work.

This is not how young people are typically encouraged to relate to adults in positions of power - parents and educators for example. They know very well that the vulgarity that they so value in their entertainment consumption is unacceptable to many authority figures:

4.M.2 There's one thing that I got off of a YouTube video. A guy called Phillip DeFranco, it was one of his videos. But anyways the thing is his message was if you're having sex with a girl without a condom then you're having sex with a... with a blank cheque. And then he quoted Kanye West saying bitches be getting pregnant on purpose, and the video ended. But it was really entertaining and it, yeah... the whole condom and blank cheque message thing is funny.

Interviewer So that's a bit different from what you got in school classes.

4.M.2 A lot different. And I highly doubt that I'd ever see [school teacher] get up in front of the whole class and say bitches be getting pregnant on purpose. (laughing) Funny, but I doubt it'd happen.

This raises difficult issues for educators who want to use entertainment education to reach young people with content that they will actually want to consume and engage with. For example, during a discussion about sex and women's periods, one group raised an internet meme they had seen on Facebook: 
19.M.4; Girls find it easy they have the best excuse, I've got my period and things like that.

19.M.3: Oh there's this picture and it's really disgusting it's got Bear Grylls and got blood all over his face and he says 'A good man loves a woman every day of the month'. And I'm like 'That's dirty'.

19.M.4; I think I saw that one.

19.M.3; That was disgusting I felt sick afterwards.

Insert figure 1 - 'A real man loves his woman every day of the month'

How do we as educators engage with a text like this? We can see that the young men in the focus group are strongly engaged with it - they delight in the play of repulsion and humour that it provokes, and they clearly remember it in the context of a discussion about sexual learning. But for many educators it would be simply revolting. Could we imagine allowing ourselves to produce something that these young men could find equally hilarious - and vulgar - that might also support them in their healthy sexual development? We know that young people dismiss information that is presented in 'scientific' language as being irrelevant to their own lives (McKee, Dore, and Watson 2014), and Cohn and Richter have recently called for sexual health information to be offered in 'lay' (which also means 'common' or 'vulgar') language that audiences recognize, are comfortable with, and that relates to their own sexual lives (Cohn and Richters 2013, 102). We agree with this call - 'vulgar' (common, rude, uneducated) forms of culture may not be to our own taste, but taking a 'culture-centred' approach to entertainment education it is clear that this is the language of our many members of our target audience, and thus we would have to have very good reasons not to embrace its communicative potential.

\section{'When I fingered my first girlfriend'}


Blokes Talking has now produced audiovisual material in the vulgar comedy genre favoured by many of the young men in our focus groups. It includes jokes across fourteen of the fifteen domains of healthy sexual development identified by McKee et al. (McKee et al. 2010) (excluded was the domain that healthy sexual development is not 'joyless', used to delineate the possible effects of child sexual abuse). Examples from two of the areas covered give a sense of the tone of the material - and in particular why it might be challenging to health educators uncomfortable with 'lay' or 'vulgar' ways of talking about sexual health. In 'Puberty', comedians reassure young men that it is normal and acceptable to be sexually aroused - but in a tone that is also vulgar, funny and recognizes the potential embarrassment of such situations:

You could be walking through Coles and see a sign saying 'Ripe Melons' and you will crack the biggest fat of your fucking life. Is there something wrong with me? No there's not. Turn that way - 'Chicken breasts' - Fuck!

The first time you saw a girl in a bikini - the school swimming carnival - a bunch of guys just hiding behind bins trying to beat their erections down with their fists.

They also reassure young men that masturbation is normal - although again, not in a preaching way, and not minimizing the potential embarrassment it can cause:

Hands up if you've ever ejaculated in your own eye. Anyone? Anyone besides me? It hurts a lot. Because all those little swimmers, they've got a journey to go on... it hurts a lot. And the worst thing is, it happened to me when I was fifteen and I had to go out and have dinner with my parents, I've got one eye all red and bleeding, mum says - 'What's wrong with your eye, Greg?'. 'I'm stoned'.

I never had a wet dream or anything like that because a wet dream is a release of a build-up and my shit never builds up. It never gets a chance. In fact sometimes I'm trying to coax it out.

'Learning about sex and relationships' covers the inadequacy of the sex education that young people receive from parents as well as school: 
I went to my father at one stage and said, 'Dad, I've got this new girlfriend'.

And I just wanted to ask him. And he's just slapped me on the back and said 'Good one son. You're really fucking her, I hope?'.

They also talked about the inadequacy of pornography as sex education:

It wasn't until the first time I fingered my first girlfriend when I was a teenager that I realized that there was a bit that it actually went in. Because all I'd seen up to that point was Playboy, which was like this hairy triangle. It wasn't until we started messing around that I realized - there's more to this than meets the eye

The comedians also provide some practical sex advice. The language used is vulgar and comedic but the advice is nevertheless sound:

The first instruction I think anyone should learn is - before you go sticking your fingers in there, make sure it's wet. Cause otherwise it's uncomfortable for everyone.

These comments illustrate the vulgar tone of the comedians, and also the way that they speak to young men in 'lay' language about sexual health issues that are important to educators, and important to young men.

\section{Conclusion}

We believe that the Blokes Talking project raises important issues for any attempt to communicate sexual health information to young people - and indeed, for health communication more generally. Health promotion materials tend to be neither ambivalent nor vulgar: yet embracing these qualities may increase their communicative potential. The use of digital means to distribute such information is an important part of embracing the vulgar and the ambivalent as formal governmental health and education institutions are likely to have a particularly difficult time being vulgar and ambivalent. By contrast, a video on YouTube is not limited by such institutional constraints. There are many obstacles still to overcome - but Blokes Talking shows that it is possible to produce material with a serious educational content that does not take itself too seriously. 


\section{Acknowledgements}

The focus groups were funded by a Queensland Government NIRAP grant "Improved Surveillance, Treatment and Control of Chlamydial Infections".

The production of the comedy footage was funded by Ansvar Insurance Community Education Fund and Andrology Australia.

\section{References}

Buckingham, David, and Sara Bragg. 2004. Young People, Sex and the Media: the facts of life? London: Palgrave Macmillan.

Byron, Paul, Kath Albury, and Clifton Evers. 2013. "'It would be weird to have that on Facebook': young people's use of social media and the risk of sharing sexual health information." Reproductive Health Matters no. 21 (41):35-44. doi: 10.1016/S0968-8080(13)41686-5.

Cohn, Amanda, and Juliet Richters. 2013. "'My vagina makes funny noises': Analyzing online forums to assess the real sexual health concerns of young people." International Journal of Sexual Health no. 25 (2):93-103.

Dutta, Mohan J. 2008. Communicating Health: a culture-centred approach. Malden, MA: Polity.

Hutcheon, Linda. 1985. A Theory of Parody: the teachings of twentieth-century art forms. London: Methuen.

Lewis, Paul. 1989. Comic Effects: Interdisciplinary approaches to humour in literature. New York: University of New York Press.

McKee, Alan, Kath Albury, Michael Dunne, Sue Grieshaber, John Hartley, Catharine Lumby, and Ben Mathews. 2010. "Healthy sexual development: an interdisciplinary framework for research." International Journal of Sexual Health no. 22 (1):14-19.

McKee, Alan, Johanna Dore, and Anne-Frances Watson. 2014. "'It's all scientific to me': focus groups insights into why young people don't apply safe sex knowledge." Sex Education no. Online first publication:1-14. doi: 10.1080/14681811.2014.917622.

Moyer-Guse, Emily, and Robin L Nabi. 2010. "Explaining the effects of narrative in an entertainment television program: overcoming resistance to persuasion." Human Communication Research no. 36 (1):26-52.

Palmer, Jerry. 1987. The Logic of the Absurd: on film and television comedy. London: BFI Publishing.

Singhal, Arvind, and Everett M Rogers. 2002. "A theoretical agenda for entertainment-education." Communication Theory no. 12 (2):117-135.

\section{Figure caption}

- Figure 1: 'A real man loves his woman every day of the month' 


\section{Disclosure statement}

This research raises no conflicts of interest for any of the authors of this article.

Length: 4818 words 